\title{
Reconfigurable Optical Filter Based on Digital Mirror Arrays
}

\author{
M. Knapczyk, A. Krishnan, L. Grave de Peralta, A. A. Bernussi, and H. Temkin
}

\begin{abstract}
We present a reconfigurable optical filter with multiple passband channels based on the combination of a diffraction grating and an array of digital micromirrors. The filter allows for adjustment of the central frequency of its individual passband channels to match standard wavelength grids with precision better than $0.033 \mathrm{~nm}$. The number of channels, the channel passband width, and the channel peak profile can be dynamically reconfigured by changing the periodic pattern imposed to the digital micromirror array. Experimental results are in excellent agreement with basic design relations describing the spectral characteristics of the filter.
\end{abstract}

Index Terms-Digital micromirrors, optical diffraction, optical filters, programmable filters.

F ILTERS with multiple passband channels are key elements in optical networks and communication systems. Multiplexers, an example of optical filters with a large number of channels, are currently used in dense wavelength-division-multiplexing systems [1]. Multiple passband filters are also used to generate pulse sequences for optical code-division multiple-access applications [2], [3]. Optical filters with reconfigurable capabilities receive considerable attention because they can provide additional flexibility to optical networks. Variable optical attenuators (VOAs) [4], dynamic optical add-drop multiplexers [5], space to wavelength switches [6], and dispersion compensators [7] are all based on reconfigurable optical filters with multiple passbands.

Several techniques have been investigated to achieve filter tunability. In particular, microelectromechanical systems have been used to construct reconfigurable multichannel filters [4]-[9]. Reconfigurable add-drop multiplexers and VOAs [5], [8], [9] based on arrays of digital micromirrors [10] have been demonstrated. The possibility of using digital micromirror arrays (DMAs) in filter applications has been discussed recently [9] but no detailed experimental results were reported. In this study, we present results of transmission output response of reconfigurable multiple passband optical filters based on DMAs. We demonstrate that the output response of the filter can be tuned to match the ITU standard grid frequencies with precision better than $0.033 \mathrm{~nm}$. This filter allows for control of the number of passband channels, wavelength separation between consecutive channels, and the spectral characteristics of each

Manuscript received March 15, 2005; revised April 8, 2005. This work was supported by the J. F Maddox Foundation.

The authors are with the Department of Electrical and Computer Engineering, Texas Tech University, Lubbock, TX 79409 USA (e-mail: ayrton.bernussi@ttu.edu).

Digital Object Identifier 10.1109/LPT.2005.850897

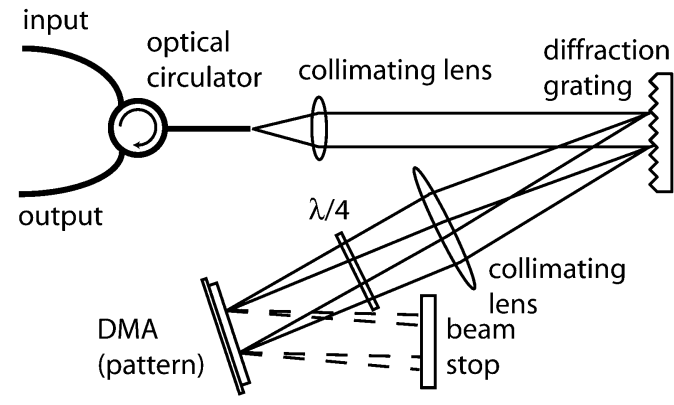

Fig. 1. Schematic diagram of the experimental setup.

passband channel. We measured negligibly small polarization dependence of the output response of the filter.

The DMA used in this study is based on a commercial display device. Individual mirrors, fabricated over static random access memory address circuit, are suspended over an air gap by two post-supported torsion hinges at opposite corners. Depending on the control signal applied to the memory cell, each mirror can be rotated by $\pm 12^{\circ}$ from the unpowered position [10]. Each of the $\sim 750000$ mirrors of the rectangular chip can be addressed independently. The mirror switching time is less than $15 \mu \mathrm{s}$. In display applications individual mirrors are reset at the video frame rate [11] in order to control hinge memory and stiction problems. In the filter experiments discussed here the resets are disabled. Recent experiments in which mirror arrays were used to implement an all-optical switch demonstrated excellent optical quality of these devices and their high mechanical stability [12].

A schematic illustration of the DMA-based reconfigurable optical filter is shown in Fig. 1. The input beam, from one arm of the fiber-optic circulator, is collimated onto a diffraction grating that disperses the frequency components at different angles. The diffracted spectrum is focused on the surface of the DMA with a lens with a focal length $f=24 \mathrm{~cm}$, spatially distributing spectral components. The spectral components of interest are selected by adjusting the ON-OFF state of groups of micromirrors arranged in lines parallel to the diagonal of the chip. These frequency components are then reflected back, collimated onto the diffraction grating by the lens, recombined, and directed to the output, using the second arm of the optical circulator. A quarter-wave plate was used to minimize polarization effects. The output response of the filter was measured with a JDS-Uniphase Swept Wavelength System (SWS-15 100) that employs the Mueller matrix analysis to determine PDL at all scanned wavelengths. The DMA-based optical filter exhibits 


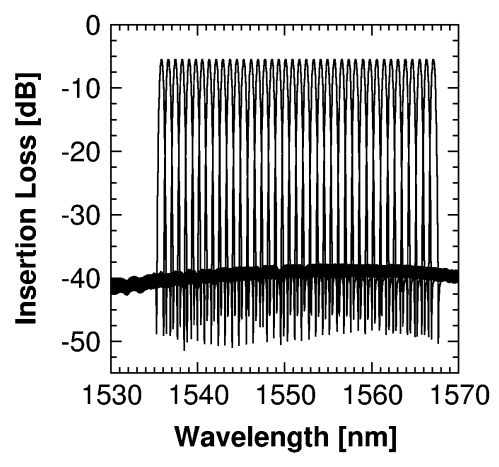

Fig. 2. Optical filter function corresponding to 40 individual passband channels separated by $100 \mathrm{GHz}$.

polarization-dependence loss of $0.75 \mathrm{~dB}$ and polarization dependent wavelength shift of $0.03 \mathrm{~nm}$. Free-space optical devices based on a combination of diffraction grating and micromirrors are known to exhibit very low chromatic dispersion [5], [13]. We, thus, focused primarily on the amplitude response of the filter.

Our experimental arrangement uses the diffraction order $m=1$ of a grating with 600 lines $/ \mathrm{mm}$ (grating period $d=1.6 \mu \mathrm{m})$ at a nominal wavelength of $\lambda=1550 \mathrm{~nm}$. With an incident angle $\theta_{\text {in }}=35.0^{\circ}$ the diffraction angle $\theta_{d}=20.88^{\circ}$ is measured. The reciprocal of the spatial dispersion $(\alpha)$ of the grating-lens combination, which describes the relative position $(x)$ of the frequency components across the diagonal of the DMA chip, is given by [2]

$$
\alpha=\frac{\partial \lambda}{\partial x}=\frac{d \cdot \cos \theta_{d}}{f \cdot m}
$$

from which we estimate $\alpha \approx 6.49 \mathrm{~nm} / \mathrm{mm}$. Micromirrors used in this work are $\sim 13 \times 13 \mu \mathrm{m}^{2}$ size with a $\sim 14-\mu \mathrm{m}$ pitch. Since the diffracted spectrum extends along the DMA's diagonal, the pitch corresponds to $\sim 9.67-\mu \mathrm{m}$ separation between consecutive micromirror lines $(D)$, or $\alpha \approx 0.063 \mathrm{~nm} /$ micromirror line. The spectral resolution of the filter is determined by the minimum size of the beam $d_{\text {out }}$ focused on the DMA when a single wavelength is used, which is given by [2]

$$
d_{\text {out }}=4 \cdot \frac{\cos \theta_{\text {in }}}{\cos \theta_{d}} \cdot \frac{f \cdot \lambda}{\pi \cdot d_{\text {in }}}
$$

where $d_{\text {in }} \sim 5 \mathrm{~mm}$ is the diameter of the input beam. Using (2), we calculate $d_{\text {out }}=0.083 \mathrm{~mm}$. In the experimental arrangement shown in Fig. 1, the beam at the surface of the DMA has an elliptical shape with the minor axis determined by $d_{\text {out }}$. This corresponds to nine lines of micromirrors. The length of the major axis is controlled by the focusing lens.

A large variety of filter functions can be implemented with the optical arrangement shown in Fig. 1. As one example, the response of the filter is adjusted to produce an output corresponding to 40 passband channels, within the $C$-band, with channel-to-channel separation of $100 \mathrm{GHz}(\sim 0.8 \mathrm{~nm})$ matching the standard ITU grid. In order to obtain this response, a pattern with a period of 12 micromirror lines, with 8 lines in the reflecting position (ON-state), was applied to the DMA. Fig. 2 shows the transmission spectra of the filter measured for one channel at a time. The 3-dB bandwidths of individual channels

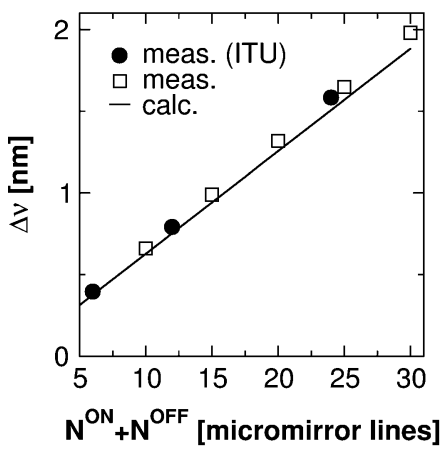

Fig. 3. Comparison between measured and calculated channel-to-channel separation as a function of the pattern period. Filled circles correspond to ITU grids of 50-, 100-, and 200-GHz channel-to-channel spacing.

are approximately $0.4 \mathrm{~nm}$ and the adjacent channel crosstalk is better than $35 \mathrm{~dB}$. Measured deviations of the individual channel peak position from the ITU grid are less than $0.033 \mathrm{~nm}$. This deviation is ultimately limited by the resolution of the DMA, the diffraction constant of the grating, and the focal distance of the lens. Measured average insertion losses in our DMA-based reconfigurable optical filter were $-6 \mathrm{~dB}$. These can be improved by using a DMA chip designed for $C$-band operation.

Spectral components of the input signal are spatially distributed over the surface of the DMA. By shifting the periodic pattern created on the DMA, different spectral components are reflected back to the output. A shift in the periodic pattern on the DMA thus results in fine-tuning of the transmission peaks' position with respect to the ITU grid. With a period consisting of 12 micromirror lines, needed for $100-\mathrm{GHz}$ channel-to-channel separation, the 40 passband channels occupy less than $5 \mathrm{~mm}$ of the $\sim 1.7-\mathrm{cm}$ diagonal length of the DMA chip. Shifting the position of the periodic pattern by one micromirror line moves the peak position of a single channel by $0.066 \pm 0.007 \mathrm{~nm}$. The shift is independent of the channel wavelength. The worst possible misalignment with respect to the ITU grid is half of that shift, namely $0.033 \mathrm{~nm}$. We take this number to represent the alignment precision of our system.

The channel-to-channel separation $(\Delta \nu)$ can be easily modified by changing the pattern period in the DMA. Channel-tochannel separation of 200 or $50 \mathrm{GHz}$ can be obtained with a period of 24 and 6 micromirror lines, respectively. In our optical system, the separation between consecutive channels is limited to $50 \mathrm{GHz}$ mainly due to the size of the beam spot on the DMA. Using (1), the values of $\Delta \nu$ in the DMA-based optical filter can be calculated as

$$
\Delta \nu=\alpha \cdot D \cdot\left(N^{\mathrm{ON}}+N^{\mathrm{OFF}}\right)
$$

where $N^{\mathrm{ON}}$ and $N^{\mathrm{OFF}}$ are the number of mirror lines/period contributing (ON) and not contributing (OFF) to the output. Fig. 3 shows a comparison between measured and calculated $\Delta \nu$. Measured channel-to-channel separation varies linearly with $N^{\mathrm{ON}}+N^{\mathrm{OFF}}$, in excellent agreement with the values calculated from (3).

The bandwidth of individual passband channels and their respective profiles depend on the number of reflecting micromirrors within each period of the pattern on the DMA. 


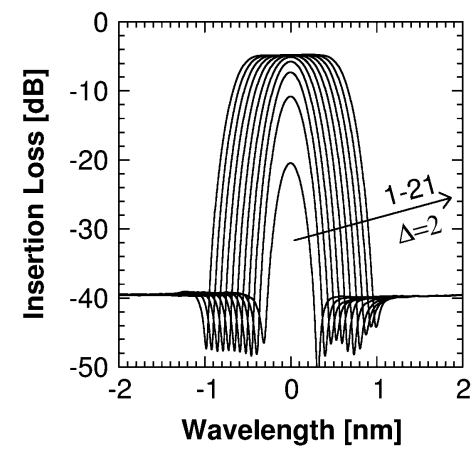

Fig. 4. Individual output channel passband as a function of number of micromirror lines in the reflective state. Number of micromirror lines was varied from 1 to 21 with a constant step of $\Delta=2$.

This allows us to tailor the transfer function of individual channels. For instance, in order to obtain a channel response with a flat-top profile, a pattern with at least nine reflecting micromirror lines/period is required. Fig. 4 shows the effect of the number of micromirror lines on the response of the individual passband channel. When the number of micromirror lines increases from 1 to 21 , the $3-\mathrm{dB}$ bandwidth of a single channel is varied from 0.233 to $1.219 \mathrm{~nm}$, respectively. The extinction ratio of an individual passband channel changes from 23 to $35 \mathrm{~dB}$, when the number of micromirror lines changes from 1 to 9 , respectively. Larger number of micromirror lines did not result in increased values of the extinction ratio. This is attributed to the size of the focused beam spot $(\sim 80 \mu \mathrm{m})$ that corresponds to approximately nine micromirror lines.

In summary, we demonstrate a reconfigurable multiple passband filter based on array of digital micromirrors. Center wavelength of individual channels of the filter can be adjusted to match the standard ITU grid with precision better than $0.033 \mathrm{~nm}$. This filter makes it possible to dynamically control the number of passband channels, peak wavelength separation between adjacent channels, and the passband profile for each channel. Basic filter design relations, including the number of micromirror lines in the pattern period, are presented and found to be in excellent agreement with experiment.

\section{REFERENCES}

[1] A. Himeno, K. Kato, and T. Miya, "Silica-based planar lightwave circuits," IEEE Sel. Topics Quantum Electron., vol. 4, no. 6, pp. 913-924, Nov./Dec. 1998.

[2] A. Weiner, "Femtosecond pulse shaping using spatial light modulators," Rev. Sci. Instrum., vol. 71, pp. 1929-1960, 2000.

[3] H. P. Sardesai, C. C. Chang, and A. M. Weiner, "A femtosecond code division multiple access communication system test-bed," J. Lightw. Technol., vol. 16, no. 11, pp. 1953-1964, Nov. 1998.

[4] J. E. Ford and J. A. Walker, "Dynamic spectral power equalization using micro-opto-mechanics," IEEE Photon. Technol. Lett., vol. 10, no. 10, pp. 1440-1442, Oct. 1998.

[5] J. E. Ford, V. A. Aksyuk, D. J. Bishop, and J. A. Walker, "Wavelength add-drop switching using tilting micromirrors," J. Lightw. Technol., vol. 17, no. 5, pp. 904-911, May 1999.

[6] D. T. Fuchs, C. R. Doerr, V. A. Aksyuk, M. E. Simon, L. W. Stulz, S. Chandrasekhar, L. L. Buhl, M. Cappuzzo, L. Gomez, A. Wong-Foy, E. Laskowski, E. Chen, and R. Pafchek, "A hybrid MEMS-waveguide wavelength selective crossconnect," IEEE Photon. Technol. Lett., vol. 16, no. 1, pp. 99-101, Jan. 2004.

[7] T. Sano, T. Iwashima, M. Katayama, T. Kanie, M. Harumoto, M. Shigehara, H. Suganuma, and M. Nishimura, "Novel multichannel tunable chromatic dispersion compensator based on MEMS and diffraction grating," IEEE Photon. Technol. Lett., vol. 15, no. 8, pp. 1109-1110, Aug. 2003.

[8] W. M. Duncan, B. Lee, P. Rancuret, B. Sawyers, and D. Powell, "DLP switched blaze grating: The heart of optical signal processing," in Proc. SPIE, vol. 4983, 2003, pp. 38-46.

[9] J. L. W. So, "Optical system and method," U.S. Patent 6842549 B2, Jan. 11, 2005.

[10] M. A. Magnardi, "From ICs to DMDs," TI Tech. J., vol. 15, pp. 56-63, 1998.

[11] H. Chu, A. Gonzalez, T. Oudal, R. Alridge, D. Dudasko, and P. Barker, "DMD superstructure characterization," TI Tech. J., vol. 15, pp. 75-86, 1998.

[12] S. Sundaram, M. Knapczyk, and H. Temkin, "All-optical switch based on digital micromirrors," IEEE Photon. Technol. Lett., vol. 16, no. 6, pp. 807-809, Jun. 2003.

[13] T. Sano, T. Iwashima, M. Katayama, T. Kanie, M. Harumoto, M. Shigehara, H. Suganuma, and M. Nishimura, "Novel multichannel tunable chromatic dispersion compensator based on MEMS and diffraction grating," IEEE Photon. Technol. Lett., vol. 15, no. 8, pp. 1109-1110, Aug. 2003. 